\title{
Developing techniques for modeling spatially heterogeneous materials
}

\author{
J.W. Leggoe \\ Chemical Engineering Department \\ Texas Tech University
}

\begin{abstract}
The macroscale properties of materials evolve under the influence of phenomena arising across a broad spectrum of length scales, with spatial heterogeneity strongly influencing processes at all length scales. In order to fully investigate the effect of spatial heterogeneity on macroscale material behavior, it is necessary to formulate models that capture the interactions between microscale material phenomena and the mesoscale linkage processes that govern macroscale behavior. In order to accomplish this, a Cellular Automata (CA) based approach has been developed, in which a material volume is divided into an array of discrete domains, with each domain being randomly assigned an individual value for a property of interest. This approach has been applied to model deformation in particulate reinforced metal matrix composites (PR MMCs) and failure in ductile aluminum alloys. The nature of spatial heterogeneity has been found to strongly influence flow stresses in PR MMCs, consistent with experimental observations. The severity of local property disparities has been found to strongly influence the variability of material response, with the effect being most pronounced at high plastic strains in ductile aluminum alloys. Future development of techniques for modeling heterogeneous materials requires the application of rigorous statistical techniques for the characterization of spatial heterogeneity in material microstructures. Quadrat and distance based techniques that show potential for characterizing microstructural heterogeneity are discussed.
\end{abstract}




\section{Computational Methods in Materials Characterisation}

\section{Introduction}

Macroscale material properties evolve under the influence of phenomena arising across a broad spectrum of length scales. As an example, ductile metallic alloys and PR MMCs typically fail via a process of void nucleation, growth, and coalescence $[1,2]$. Damage initiates at the microscale in the form of voids, which nucleate preferentially at discontinuities such as secondary (or reinforcing) phases, grain boundaries, and processing flaws. As straining proceeds, voids grow and coalesce to form a population of microcracks. Final failure ensues from the linkage of microcracks to form a failure path spanning the entire specimen.

At each length scale, spatial heterogeneity strongly influences deformation and failure. The ease of void nucleation and void size are affected by the nature of the initiating defects and the spatial distribution of void initiation sites. In silicon-rich aluminum alloys, stress concentration within clustered silicon particles promotes void nucleation by particle fracture [3]. In PR MMCs, particle clusters are favored sites for void nucleation, due to both the increased population of potential nucleation sites, and the complex strain fields arising between closely spaced particles [2].

Void coalescence is in turn strongly influenced by void size and spacing. For spheroidized carbon steels, the average void length at coalescence has been found to correlate approximately with the spacing of the nucleating cementite particles [4]. Similarly, in a silicon carbide particle reinforced aluminum matrix PR MMC, decreasing particle spacing was found to decrease the void content at the onset of fracture [5]. The extent of void growth prior to coalescence is also likely to be sensitive to factors such as void size, the local stress state, and the nature of the material in the ligament separating adjacent voids.

The linkage of microcracks will vary in difficulty according to the arrangement of microcracked regions. If a convenient linkage path exists, failure may arise with little additional straining beyond the onset of void coalescence [4]. In silicon-rich aluminum alloys, interdendritic segregation creates a network of clustered silicon particles, providing an easy path for crack propagation [3]. If, however, microcracked regions are separated by defect free material, significant additional straining may be required to rupture the intervening ligament [6].

The nature of spatial heterogeneity may therefore profoundly influence macroscale material properties. Experimental data indicate that the ductility and fracture toughness of PR MMCs are significantly reduced under the influence of particle clustering $[7,8]$. In contrast, PR MMC yield strength is significantly increased by reinforcement clustering [7].

Systematic experimental investigation of the effects of spatial heterogeneity is hindered by the difficulty inherent in controlling heterogeneity during specimen preparation. Numerical modeling provides an alternative whereby sets of statistically equivalent model "specimens" can be created and analyzed. One approach is to use "expanded cell", or "multi-particle" (MP) models, in which a region of material containing multiple randomly located discontinuities is considered. 2D MP models have been used to investigate the transverse properties of unidirectional fiber-reinforced composites [9]. For ductile alloys and 
PR MMCs, the 3D arrangement of discontinuities necessitates the use of 3D models [10]. 3D-MP models of PR MMCs have been developed to investigate the influence of reinforcement clustering on the development of flow stresses and particle fracture during elastic and early plastic straining [11,12].

To date, MP models have considered relatively small domains containing on the order of 40-50 reinforcement particles. To fully investigate the effects of spatial heterogeneity on macroscale response a Cellular Automata (CA) approach has been adapted to model heterogeneous materials. The application of the CA approach to model deformation in PR MMCs and failure in ductile aluminum alloys is discussed in section 2 .

A critical concern in the development of models of spatially heterogeneous materials lies in ensuring that the simulated microstructures accurately represent real material microstructures. Invariably, heterogeneity is introduced in models through the random dispersion of particles or the random assignment of properties. Real material microstructures are rarely truly random, definite patterns developing under the influence of the physical processes occurring during fabrication. In section 3, statistical approaches to the characterization and subsequent simulation of microstructures are introduced.

\section{Cellular Automata based models of heterogeneous materials}

CA were initially developed by Von Neumann [13], motivated by an interest in biological reproduction. Conventional CA consist of discrete spatial lattices, in which each element of the lattice can take one of a set of discrete values. After the assignment of initial values, the lattice evolves over discrete time steps, lattice values being changed according to the values in surrounding elements in line with rules or look-up tables. While the CA approach has commonly been applied to model problems in economics and disease propagation, interactions between disparate adjacent regions are also critical to the evolution of material behavior.

For a CA based material model, a material volume is divided into discrete domains, with each domain being assigned an individual value for the variable property of interest. A 3D CA approach was initially developed to model deformation in PR MMCs [10]. Conventional unit cell models were used to generate constitutive models for PR MMCs having varying reinforcement volume fraction. CA models were then formulated in which domain volume fractions were randomly assigned in line with either a Gaussian governing distribution or a distribution representing extreme reinforcement clustering. Figure 1(a) depicts a model representing extreme reinforcement clustering, in which each domain was randomly assigned a volume fraction of either 0 or $40 \%$. Figure 1(b) depicts a model in which domain volume fractions were assigned in line with a Gaussian distribution having a mean of 0.2 and a standard deviation of 0.1 .

The effect of spatial heterogeneity on strain field evolution in a model representing extreme reinforcement clustering is depicted in figure 2. During early straining, the strain field, while locally non-uniform, was approximately homogeneous over the entire model, with no region being particularly distorted. With continued straining, plastic strain localized within grouped weak domains, 
148 Computational Methods in Materials Characterisation

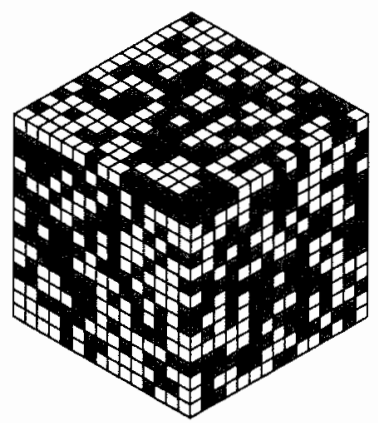

(a)

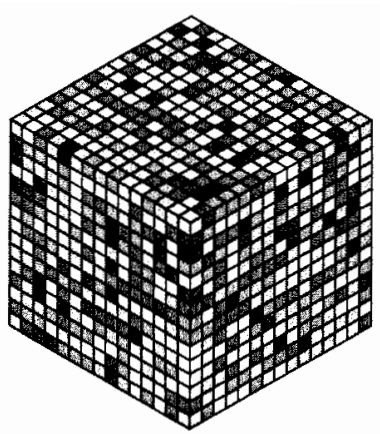

(b)

Figure 1: $\quad$ CA models of PR MMCs having spatially varying reinforcement volume fraction. (a) Model representing extreme reinforcement clustering; light elements are unreinforced, dark elements contain $40 \%$ reinforcement volume fraction. (b) Model assigned domain volume fractions in line with a Gaussian distribution having a mean of $20 \%$ and a standard deviation of $10 \%$; element colors darken progressively as volume fraction increases from 0 to $40 \%$

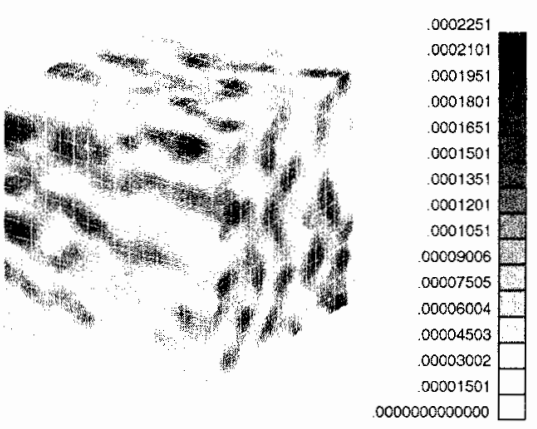

(a)

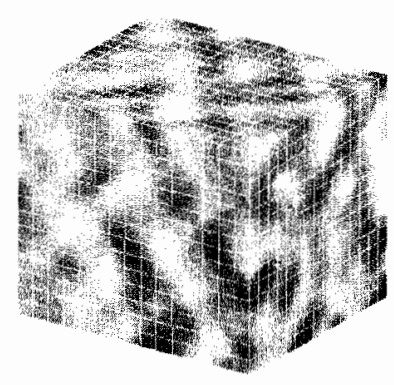

(b)

Figure 2: $\quad$ Strain field evolution in a CA model representing reinforcement clustering in a PR MMC (model (a) in figure 1). (a) Equivalent plastic strain field at an applied strain of 0.0025. (b) Equivalent plastic strain field at an applied strain of 0.10 . 
although, in the absence of a damage mechanism, gross strain localization did not arise. The severity of local property disparities strongly influenced strain field evolution: clustered models gave rise to much larger ranges of plastic strain than did the more uniform Gaussian models. Yield strength was found to increase significantly as the severity of reinforcement clustering increased, in keeping with experimental observations.

The availability of a constitutive model representing damage accumulation for aluminum alloys permitted the application of $\mathrm{CA}$ based models to investigate the mesoscale linkage processes leading to final failure. In this case, spatial heterogeneity was introduced by varying the initial porosity throughout cylindrical specimens. Initial investigations focused on determining the effect of levels of domain and property discretization on CA model predictions [14]. While the predicted ultimate strength converged for a $2 \times 2 \times 2$ domain discretization, a $4 \times 4 \times 4$ discretization was necessary to predict behavior at high strains. With the onset of final necking, it was noted that the stress supported by the specimen became strongly dependent on the element size. If CA are to be used to model final failure, care must be taken to ensure that element dimensions and failure strains reflect the volume of material participating in the real necking process.

Subsequent investigations focused on determining the influence of the nature of spatial heterogeneity on macroscale material response [15]. Three probability distributions for initial domain porosity were considered; "Uniform", where there is an equal probability of selecting each of a set of eleven initial porosity values between 0 and 0.1 ; "Extreme", where domains may take initial porosities of either 0 or 0.1 ; and "Gaussian", where the initial porosity was assigned in line with a Gaussian governing distribution with a user-defined mean and standard deviation. For each of the distributions under consideration, a set of 20 models was run.

As the spatial distribution of initial porosity deviated from the homogeneous ideal, the strain field became increasingly heterogeneous, the effect being most pronounced at high strains as strain localization developed. Figure 3 compares the strain fields in models assigned extreme and Gaussian distributions; the more uniform Gaussian distribution gave rise to a more regular strain field, strain localizing almost symmetrically about the specimen mid-section. Once again, increasing the severity of local property disparities increased the range of observed strains; the peak strain in the clustered specimen was almost double that in the Gaussian specimen. A unique strain localization path developed in each model, regardless of the distribution governing initial porosity assignment.

The effect of spatial heterogeneity on the macroscale stress-strain responses is presented in figure 4. As the initial porosity distribution deviated from the heterogeneous ideal, the variability of the macroscale response increased, with variation maximized in models representing severe porosity clustering. Variation was most pronounced at elevated strains, where the strain localization path exerted a strong influence on the macroscale response. With increasing strain, the average stress supported by the models declined significantly as the initial porosity distribution deviated from the homogeneous ideal, indicating that the severity of local property disparities plays an important role in the development of strain localization. 
150 Computational Methods in Materials Characterisation

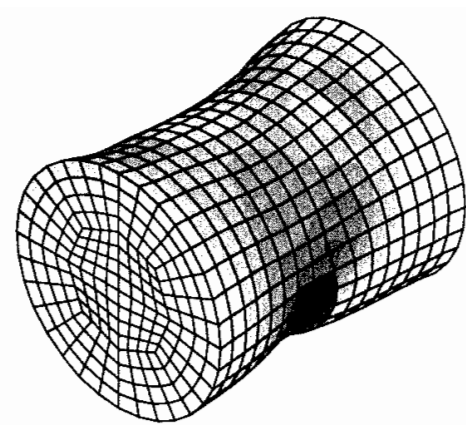

(a)

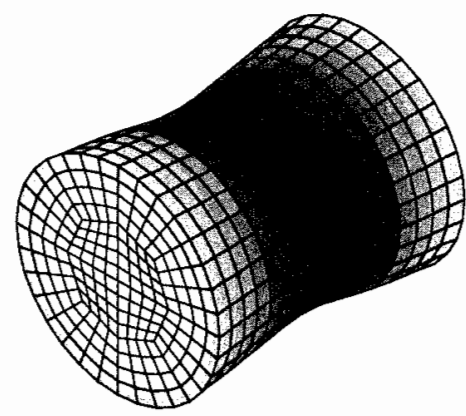

(b)

Figure 3 Effect of the nature of spatial heterogeneity on strain field evolution. Contours represent axial strain; colors darken progressively with increasing strain. (a) Model representing extreme clustering of initial porosity; peak strain is 0.562 . (b) Model in which initial porosity was assigned in line with a Gaussian distribution with standard deviation 0.01 ; peak strain is 0.310 [19].

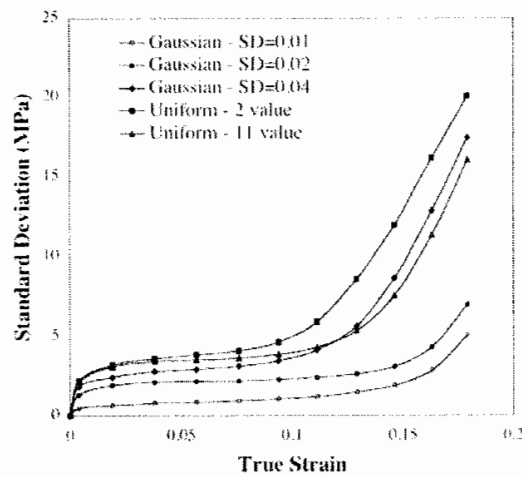

(a)

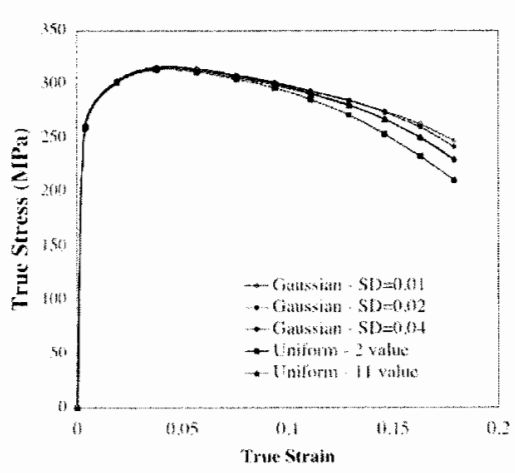

(b)

Figure 4: Effect of the nature of spatial heterogeneity on material response in sets of 20 models. (a) Averaged macroscale true stress-true strain response (b) Standard deviation in macroscale stress-strain responses [19]. 


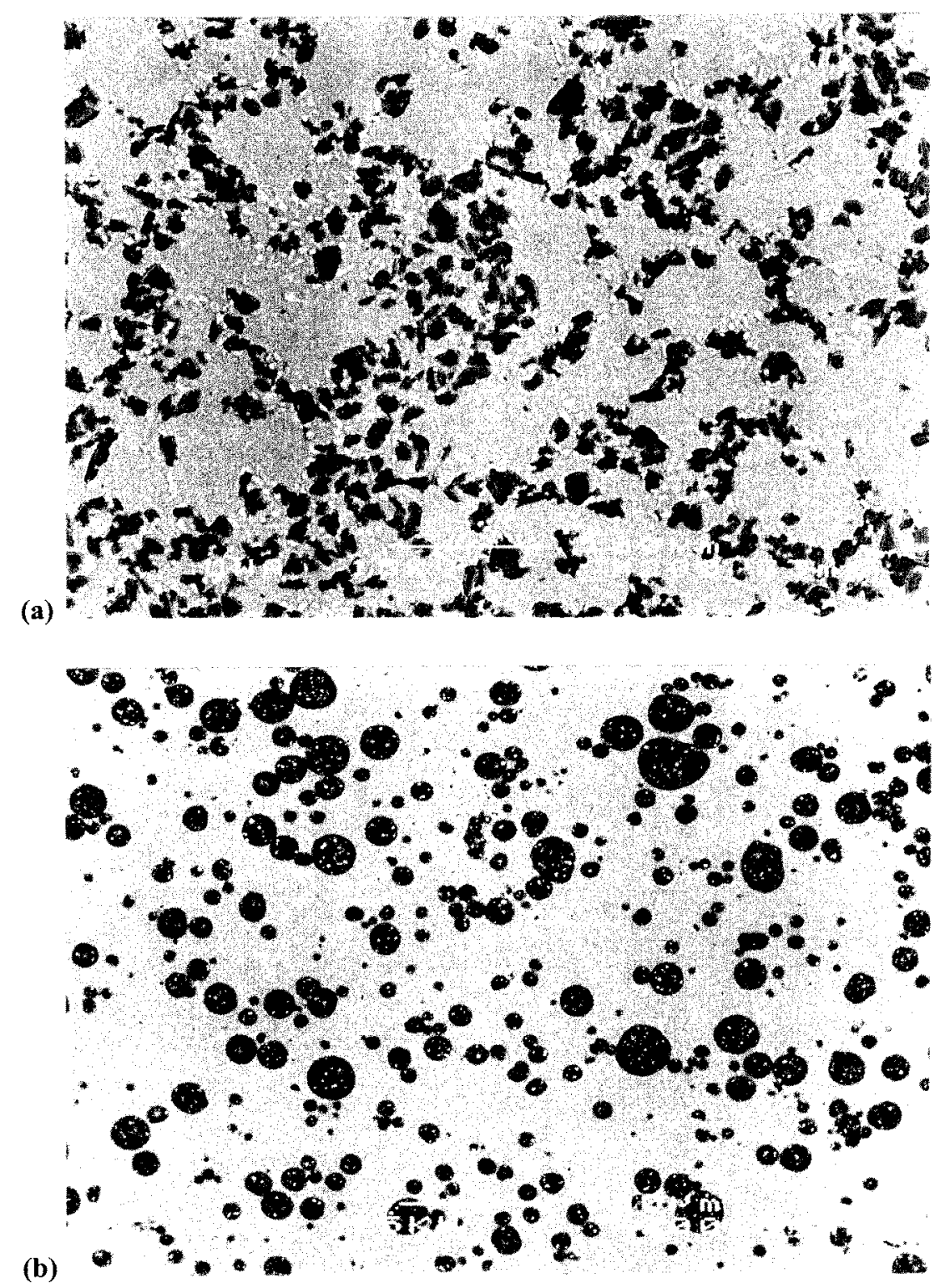

Figure 5: Particulate reinforced metal matrix composites exhibiting different degrees of reinforcement clustering; (a) Duralcan F3S.20S, aluminum $359-20 \%$ silicon carbide, in as cast state. (b) Comral$85^{\mathrm{TM}}$, aluminum $6061-20 \%$ Micral $^{\mathrm{TM}}$ microspheres, cast and extruded. 


\section{Computational Methods in Materials Characterisation}

\section{Characterization of spatial heterogeneity in materials}

One of the critical challenges in modeling heterogeneous materials lies in creating simulated microstructures that are equivalent to real microstructures. Models representing spatially heterogeneous materials have usually been generated under an assumption of complete spatial randomness (CSR). In CA models, domain properties have been randomly assigned [10,14-15], while in MP models particle centers are usually randomly dispersed $[9,12]$; when clustering has been simulated in MP models, the cluster centers have been randomly dispersed [11].

In practice, real microstructures evolve under the influence of physical processes, and are rarely truly random. Consider the PR MMC microstructures presented in figure 5. The Duralcan material exhibits interdendritic segregation of the reinforcement; In the Comral $-85^{\mathrm{TM}}$ material, extrusion has homogenized the microstructure, though there is "apparently" some residual clustering. "Apparently" recognizes a key difficulty in characterizing spatial heterogeneity; for even random spatial processes there is a significant probability that nearest neighbor spacing will be near zero [16], giving rise to "apparent" clustering.

In modeling spatial heterogeneity, methods by which intuitive concepts such as "randomness" and "clustering" can be mathematically characterized and reproduced are essential. Ideally, those techniques should also identify the length scale associated with any spatial patterns (such as particle clusters). It must also be recognized that multiple levels of heterogeneity are present in real materials; in addition to recognizing the spatial pattern of particular events (such as particle centers), variation in particle size and/or orientation may also be significant.

An excellent review of statistical techniques for characterizing spatial data is provided by Cressie [16]. Many problems in microstructural characterization require characterization of "spatial point processes", with stochastic models being sought to describe the location of "events" (for example, the location of particle centers) in a two- or three-dimensional space. "Marked" spatial point processes, in which measures associated with an event (such as particle diameter) are themselves variables, are also commonly encountered.

In "quadrat" techniques, a study region is divided into subspaces; for example, a micrograph may be divided into a regular grid of square quadrats. The number of events within each quadrat is counted, enabling the compilation of a frequency distribution for the number of events per quadrat. For a CSR process, the number of events per quadrat has a Poisson distribution with mean $\lambda A_{1}$, where $\lambda$ is the intensity of the distribution, and $A_{1}$ the quadrat area [16].

If a process fails a conventional chi-square test for CSR, a variety of indices are available to quantify the extent of clustering. Of these indices, the DavidMoore index [17] and the Index of Cluster Frequency [18] appear to offer the most potential for quantifying cluster dimensions and spacing, a critical consideration for the formulation of CA style models. The David-Moore index offers the additional capability of identifying regularity in the event pattern [16].

Quadrat techniques have the disadvantage that the results are sensitive to, and provide information only on the length scale of, the quadrat dimensions. Distance methods, in contrast, provide information across a range of length scales. The 
simplest of these, the nearest-neighbor method, simply analyzes the spacing of events and their nearest neighbors. In addition to providing a test for CSR, the average nearest neighbor spacing can provide an indication as to the "intensity" of clustering in the event that clustering is indicated.

Extended nearest neighbor methods, which consider the spacing to the "kth" nearest neighbor, have the potential to provide a clear indication of both the existence of clustering and the cluster size. From a given map of events, it is possible to compute the distance $W_{k}$ to the $k$ th nearest neighbor of each event, and thence compute the mean value of $W_{k}$. The ratio $R_{k}$ of this mean to the mean expected under CSR may then be computed. For CSR, the ratio should be one, whereas if the ratio is less than one, clustering is indicated [16]. Plotting $R_{k}$ as a function of $k$ provides a clear indication as to the extent of individual clusters.

Once a spatial point process has been characterized, a variety of simulation techniques are available. A typical simulation will randomly generate "parent events", and then allow each parent to produce a number of offspring in line with a density function. The offspring events then compose the final (simulated) spatial point process. Segurado et al [11] adopted such an approach to represent clusters in PR MMCs, each parent cluster containing a fixed number of particles. In the more general case, the number of offspring would be allowed to vary randomly in line with a governing frequency distribution, the parameters of which could be determined using extended nearest neighbor analysis of the microstructure. If the pattern exhibits regularity, "inhibition" processes such as Markov point processes may be used to simulate the structure. Simulating a marked spatial point process may also be accomplished, if given the characteristics of the marking property. Care must be taken to identify nonstationarity in the marking property; local particle alignment, for example, may strongly influence properties, and should ideally be represented in any model.

\section{Conclusions}

CA based approaches show potential for modeling the macroscale behavior of spatially heterogeneous materials, capturing the evolution of behavior under the influence of processes arising across the full range of significant length scales. The continuing development of models representing spatial heterogeneity requires the application of spatial statistical techniques to rigorously characterize spatial heterogeneity in material microstructures. Both quadrat and distance methods offer techniques by which it may be definitively determined whether a material microstructure exhibits CSR or some form of clustering. Extended nearest-neighbor models exhibit particular potential for characterizing clustering.

\section{References}

[1] Ashby, M.F., Gandhi, C., \& Taplin, D.M.R., Fracture mechanism maps and their construction for F.C.C. metals and their alloys, Acta Metallurgica, 27, pp 699-729, 1979 
154 Computational Methods in Materials Characterisation

[2] Leggoe, J.W., Hu, X.Z., \& Bush M.B., Crack tip damage development and crack growth resistance in particulate reinforced metal matrix composites, Engineering Fracture Mechanics, 53, pp 873-895, 1996

[3] Caceres, C.H., Davidson, C.J., \& Griffiths, J.R., The deformation and fracture behaviour of an Al-Si-Mg casting alloy, Materials Science \& Engineering, A197, pp 171-179, 1995

[4] Le Roy, G., Embury, J.D., Edwards, G., \& Ashby, M.F., A model of ductile fracture based on the nucleation and growth of voids, Acta Metallurgica, 29, pp 1509-1522, 1981

[5] Mummery, P.M., Anderson, P., Davis, G.R., Derby, B., \& Elliot, J.C., Damage assessment in particle reinforced metal matrix composites using $\mathrm{X}$ ray microtomography, Sctipta Metallurgica et Materialia, 29, pp 14571462, 1993

[6] Lloyd, D.J., Aspects of fracture in particulate reinforced metal matrix composites, Acta Metallurgica et Materialia, 39, pp 59-71, 1991

[7] Murphy, A.M., \& Clyne, T.W., The effect of initial porosity and particle clustering on the tensile failure of cast particulate Proceedings ICCM-10, eds K.Street, A. Poursatip Whistler, British Columbia, 1995.

[8] Roebuck, B., \& Lord, J.D., Plane strain fracture toughness testing procedures for particulate metal matrix composites, Materials science and Technology, 6, pp 1199-1209, 1990

[9] Brockenbrough, J.T., Suresh, S., \& Wienecke, H.A., Deformation of metalmatrix composites with continuous fibres: Geometrical effects of fibre distribution and shape, Acta Metallurgica et Materialia, 39, pp 735-752, 1991

[10] Leggoe, J.W., Mammoli, A.A., Bush, M.B., \& Hu, X.Z., Finite element modeling of deformation in particulate reinforced metal matrix composites with random local microstructure variation, Acta Materialia, 46, pp 60756088,1998

[11] Segurado, J., Gonzalez, C., \& Llorca, J., A numerical investigation of the effect of particle clustering on the mechanical properties of composites, Acta Materialia, 51, pp 2355-2369, 2003.

[12] Shen, H., \& Lissenden, C.J, 3D finite element analysis of particle reinforce aluminum, Materials Science \& Engineering A338, pp 271-281, 2002

[13] Von Neumann, J., Theory of Self-Reproducing Automata, University of Illinois, Urbana, Illinois, 1966

[14] Khvastunkov, M., \& Leggoe, J.W., Adapting a Cellular Automata Approach to Model Ductile Failure in Spatially Heterogeneous Metallic Alloys, submitted to Computers \& Structures, January 2003

[15] Khvastunkov, M., \& Leggoe, J.W., Modeling the influence of the nature of spatial heterogeneity on the deformation and failure of porous ductile alloys, submitted to Materials Science \& Engineering A, May 2003

[16] Cressie, N.A.C., Statistics for Spatial Data, Wiley, New York, 1991

[17] David, F.N., \& Moore, P.G., Notes in contagious distributions in plant populations, Annals of Botany, 18, pp 47-53, 1954

[18] Douglas, J.B., Clustering and aggregation, Sankhya B, 37, pp 398-417, 1975 